\title{
La dominancia social: una aproximación primatológica 1
}

\section{Fernando Peláez *}

Universidad Autónoma de Madrid y Parque Zoológico de la Casa de Campo de Madrid

El concepto de dominancia ha sido y continúa siendo uno de los temas de debate más controvertidos en el estudio de la conducta social. La dominancia ha tenido un enorme interés, tanto por el valor del concepto mismo como por su significado sociológico en ambientes científicos y extracientíficos. Máxime tras la popularización hecha por algunos autores con la intención de "explicar» nuestras tendencias naturales (Ardrey, 1967).

Un animal se considera dominante cuando en sus interacciones «domina» sobre otro (Rowell, 1974), y se habla de. jerarquía de dominancia cuando en un grupo social los individuos ócupan distintas posiciones o rangos dependiendo de su dominancia relativa sobre el resto de los individuos.
Desde que Schjelderupp-Ebbe, en I 922 , postulara el concepto de «dominancia social», se han producido muchas polémicas en torno a su definición, valor descriptivo y explicativo, función de la dominancia y validez del concepto mismo.

Estudiando la gallina doméstica, Schjelderupp-Ebbe afirmó que los individuos podian ordenarse linealmente conforme a sus interacciones sociales. Estas interacciones consistían en que algunos animales picaban a otros de forma que, en cada interacción diádica, la dirección del picoteo era unidireccional: desde el "déspota» al "sumiso". El criterio utilizado para establecer este orden ("orden de picoteo») se basaba únicamente en la agresividad exhibida por determinados individuos en la interacción y que se manifestaba me-

1 Este artículo se basa en la tesis doctoral del autor, dirigida por el profesor doctor don Arsenio Fraile Ovejero (catedrático de Fisiología Animal de la Facultad dé Ciéncias Biológicas de la Universidad Complutense de Madrid), a quien quiero expresar mi más sincero agradecimiento.

José E. Ortega Seyó, Margarita Toral corrigió y mecanografió el manuscrito, a ella le debo todo.

Por último, quiero agradecer a la Dirección del Parque Zoológico de la Casa de Campo de Madrid su amabilidad por permitirme el acceso a sus instalaciones desde el año 1974.

La tesis doctoral de la que este artículo forma parte fue financiada por la Caja de Ahorros y Monte de Piedad de' Madrid, a través del préstamo concedido al autor.

* Dirección del autor: Dpto. de Psicobiologia, Facultad de Psicología, Universidad Autónoma. Campus de Cantoblanco. 28049 Madrid. 
diante picar. Es decir, la dominancia era unidimensional, ya que se utilizaba un único criterio (picar) para establecer la jerarquía por la que se regulaba la estructura social del grupo (Syme, I 974).

Por otra parte, la dominancia era considerada como una cualidad, inherente a algunos animales, de la que carecian los individuos sumisos (Zuckerman, 1932; Maslow, I936a y 1940). Esta visión antropomórfica hacia ver a los animales dominantes como poseedores de un alto impulso para la dominación, aunque no se llegara a plantear que los sumisos poseyeran un alto impulso para la sumisión (Maslow, i 936b; Rowell, 1974).

El concepto de dominancia social fue utilizado en todos los vertebrados, excepto peces y anfibios (Crawford, 1939 cit., en Syme, 1974). La jerarquía se consideraba inicialmente como el principio básico de organización de la estructura social de los animales que vivian en grupos, incluyendo a los primates (Noë et al., 1980 ).

\section{UNIDIMENSIONALIDAD DE LA DOMINANCIA}

La primera objeción a la unidimensionalidad de la dominancia proviene del modelo mismo. Por lo general, la dominancia se habia estudiado en condiciones de libertad, en momentos en los que los investigadores carecían de la suficiente documentación que les permitiera utilizar el mayor número de conductas sociales. Cuando la dominancia se estudiaba en grupos formados artificialmente, la agresión de los primeros momentos tendía a desaparecer cuando el grupo se establecía.

Posiblemente, debido a la existencia de un orden jerárquico estable en libertad o por el asentamiento del mismo después de la formación de los grupos en cautividad, las agresiones abiertas llegaban a ser tan difíciles de observar que resultaban una mala medida o al menos insuficiente para determinar una jerarquía de dominancia. Este hecho era contradictorio con la unidimensionalidad y hacía pensar en la existencia de otros principios determinantes. De cualquier forma, los nuevos criterios que se consideraron estaban muy relacionados con la agresividad misma, no haciendo más que complementarla.

La jerarquía de dominancia se estableció a partir de criterios como el de ser ganador/perdedor en luchas (Sade, 1967; Varley y Symmes, 1966), de la prioridad de acceso a incentivos como alimento (Imanishi, I960; Bernstein y Sharpe, 1966), lugar y hembras (Chance, 1956; DeVore, 1965). También se consideró el éxito en el apareamiento (Hall y DeVore, 1965; Hausfater, 1975), conductas agonísticas en general (Tokuda y Jensen, 1969) y conductas de aproximación/separación (Rowell, 1966 y 1967).

Sade (1967) propuso una jerarquia de dominancia basada en criterios agresivos cuando estudió una tropa de thesus (Macaca mulatta) en Cayo Santiago. «Dado que algunas conductas como monta, espulgamiento, bostezo, etc., pueden continuarse con las mismas conductas por parte del otro primate durante la interacción...» (pág. 95), Sade empleó los criterios derivados de la agresión misma, es decir, ganadores/perdedores en una confrontación agonística.

Hall y DeVore ( 1965 ) encontraron una alta correlación entre la cantidad de apareamiento realizado por los machos y su rango de dominancia establecido mediante otros criterios agonísticos. Así, en el caso de babuinos chacma (Papio ursinus) en libertad, puede añadirse un nuevo criterio de dominación correlacionado con los anteriores.

Rowell (1966 y 1967) estudió un grupo de babuinos oliva (Papio anubis) de ambos sexos en cautividad, estableciendo un "rango aparente» (medida arbitraria del rango obtenida por simple intuición) a partir de criterios comúnmente utilizados: prioridad en la alimentación, elección de lugares preferentes, etc. Después de comparar con los datos reales obtenidos tras la observación de un conjunto de conductas agresivas y no agresivas, Rowell ( 1966 ) 
reunió estas conductas en tres categorías que definió como: aproximación/separación no agonísticas, aproximación/separación agonísticas y amistosas. Del conjunto de las observaciones concluyó que la dominancia podía establecerse en la medida en que las interacciones sociales respondan o no al patrón "A se aproxima a $\mathrm{B}$ ». El resto de las interacciones ( 72 por Ioo) correspondian a interacciones amistosas. Rowell observó que ningún patrón sencillo de conducta podía utilizarse como criterio para establecer un rango de dominancia, ya que, incluso algunos patrones de conducta que resultaban correlacionados con el rango aparente de los machos carecían de correlación considerados con el rango aparente de las hembras. Por tanto, sólo el criterio de aproximación/separación era válido para establecer una jerarquía. Si conductas tales como la agresión directa se hacen inutilizables para formar un orden jerárquico de dominancia, Rowell estableció categorías más altas a partir de patrones más sencillos, considerando tanto interacciones agresivas como las que no lo eran. El criterio de aproximación/separación también ha sido utilizado por Simonds (1965) y Kaufmann ( 1967 ) entre otros.

Como se puede ver, todos los criterios utilizados para el establecimiento de una jerarquía de dominancia son derivados de la agresión o son un reflejo de la agresión misma. Si se admitía la unidimensionalidad, dado que agresión, aproximación/separación, prioridad, etc., son representativos del mismo fenómeno, podían establecerse un conjunto de órdenes competitivos que sustituyesen a los métodos de observación, respecto a un conjunto de variables controladas. Los órdenes competitivos se establecían en situaciones de control artificial y pueden considerarse como un método sustitutivo de los órdenes de dominancia. No obstante (Syme, I974), el problema seguiría siendo el mismo que para los órdenes basados en la dominancia, si el criterio de unidimensionalidad no fuese adecuado. Además, no tenía por qué haber necesariamente una correlación entre los órdenes de do- minancia basados en la agresión y los órdenes competitivos, al menos entre los distintos grupos taxonómicos.

Varley y Symmes (1966) encontraron una correlación positiva entre prioridad ante el alimento o a otros incentivos y la interacción agonística en macacos, pero Reynolds y Luscombe (1969) no pudieron correlacionar agresión y prioridad en chimpancés. Kaufmann (1965, citado en Richards, 1974) pudo correlacionar éxito en el apareamiento con rango agresivo en macacos rhesus, pero Jolly (1967) no halló los mismos resultados en otras especies. Para Kaufmann (1967) la amenaza y la monta correlacionaban con el rango jerárquico agresivo; pero Bernstein (1970), estudiando seis especies de primates encontró que cinco de ellas poseían jerarquías estables con criterios basados en interacciones agonísticas, aunque en términos de monta las jerarquías eran menos claras y menos aún si consideraba el espulgamiento. Bernstein concluyó que estas tres respuestas no se derivaban de un único mecanismo social.

Por otra parte, el concepto de dominancia se complicaria más si dentro de cada grupo social considerásemos las distintas clases de edad/sexo. Para Rowell (1966) la jerarquía debería encontrarse separadamente para cada una de las distintas clases de edad/sexo, porque en cada una de ellas hay una cierta homogeneidad en las conductas. Pero como las interacciones dentro del grupo ocurren entre y a través de las distintas clases, la división puede producir alteraciones que no correspondan con la estructura social real del grupo. Sade (1967) separó cada una de las clases de edad/sexo y afirmó que dentro de cada una, la jerarquía de los individuos de acuerdo a sus luchas ganadas y perdidas, era lineal. Algunos autores han considerado también que los órdenes de dominancia y los criterios para establecerlos no son coincidentes, al menos para las clases de sexo (Hall y DeVore, 1965 ; Lee y Oliver, 1979), pero otros han ordenado a todos los individuos en una sola jerarquia (Struhsaker, 1967). Colocar a todos juntos tiene el problema de que se amal- 
gaman diferencias competitivas entre las distintas edades y los distintos sexos; pero el separarlos es considerar que el conjunto del grupo no es un todo (Pollock, 1979; véase también Lee y Oliver, 1979).

A modo de síntesis de los distintos elementos hasta ahora vistos, la utilización de un único criterio no era una forma útil con la que establecer una jerarquía de dominancia. Los intentos de encontrar nuevos criterios han caído en ser un complemento de aquéllos basados en la agresión misma. Utilizando criterios distintos de éste se observó que no resultaba fácil encontrar correlaciones entre ellos, por lo que las jerarquías establecidas no siempre eran coincidentes. Por otra parte, aparecían dificultades al establecer las jerarquias del grupo, tanto al considerar las clases de edad/sexo en su conjunto como al obtener un rango por separado para cada una de ellas. Cuando se evaluó el poder de jerarquización que los distintos criterios poseían, se comprobó que no siempre aparecían correlaciones válidas para los distintos grupos taxonómicos ni, incluso, para grupos de la misma especie que no compartían los mismos ambientes.

Como consecuencia, algunos autores buscaron términos que sustituyesen al de dominancia. En este sentido se propuso que en una sociedad existían una variedad de «roles» relacionados entre sí y que existian señales que indicaban especificamente dichos papeles actuales o potenciales en los individuos. «La complejidad de los patrones de interacción asociados con un rol y el número de roles distintos en el grupo pueden estar relacionados con el grado de complejidad social. De esta forma las sociedades pueden compararse mediante un análisis detallado de los roles característicos de cada una de ellas. Los roles vienen determinados en los humanos $\mathrm{y}$ en los primates no humanos por factores biológicos y sociológicos: edad, sexo y estatus" (Bernstein y Sharpe, 1966, pág. 91). Posteriormente, Gartlan (1968) y Crook (1970) aconsejaron la búsqueda de perfiles de un papel social que sustitu- yese al, según ellos, inadecuado término de dominancia. Sin embargo, el concepto de rol utilizado por los sociólogos implicaba una abstracción de normas de comportamiento. El rol no depende del individuo o individuos que lo ostentan. Desde el punto de vista de Gartlan, el concepto de rol podía ser de utilidad al analizar la significación adaptativa para el grupo en su conjunto, pero no decía nada de las dinámicas intragrupales (Hinde, 1974). En su utilización, rol ha sido confundido con conducta $y$, por tanto, carece de valor como sustituto de la dominancia. Rol y dominancia plantearian los mismos problemas si se tratasen de esta forma (véase Hinde, I974 y 1978).

También con la intención de obtener un mejor concepto que sustituyese al de dominancia, M. R. A. Chance propuso el modelo de ula estructura social de la atención». El rango social podia encontrarse en función de la cantidad de atención recibida por algunos individuos de parte del resto del grupo (Chance, I 967). Mediante este nuevo criterio se explicaba la estructura social de los distintos grupos de primates, que podían ser clasificados dependiendo del tipo de estructura de atención que poseyeran. El modelo pretendia ser conceptual, de forma que sustituyese al de dominancia. El problema era que el argumento se convertía en circular cuando se utilizaba la atención para establecer qué animal ocupaba el primer lugar en el orden de rango, pasándose posteriormente a considerar a algunos animales como dominantes en función de la mayor cantidad de atención recibida (Torres de Asunçao y Deag, 1979). El estudio de la atención podía ser útil como un elemento descriptivo más de la estructura social, pero no tenía que ser considerado el principal y mucho menos el único (Hinde, 1974). Por otra parte, al estudiar la atención (medida por la dirección de la mirada) y la jerarquía de dominancia en términos de interacciones agonísticas, Torres de Asunçao y Deag (1979) no encontraron ninguna correlación que apoyase la sustitución de un término por otro. El principal obs- 
táculo sería la definición misma de la atención y las dificultades de cuantificarla en la práctica (para una discusión más amplia del concepto de «estructura de la atención" puede verse Chance y Jolly, 1970; Chance y Larsen, 1976; Emory, 1976, y Chance, 1978).

Por último, se ha puesto en cuestión el valor de la dominancia como elemento descriptivo, en el sentido de que la interacción pudiera estar determinada no por el animal dominante, sino por el papel que jugase el subordinado. El animal subordinado mantendría actitudes constantes hacia el dominante, pudiendo definirse a este último como uel que no piensa antes de actuar) (Rowell, I 966 y 1974). Mason (1961) también indicó que las acciones del subordinado podian ser las que determinasen la existencia de una jerarquia de dominancia y el mismo Chance $(1967)$ consideró que la mayor atención que recibian los dominantes era independiente de su actitud y dependiente de la de los subordinados (véase también Rowell, 1966 y i 974).

\section{UNIDIRECCIONALIDAD Y ESTABILIDAD DE LA DOMINANCIA SOCIAL}

Schjelderupp-Ebbe había descrito la dominancia en términos de interacciones sociales. Posteriormente se buscó una definición en función de sus consecuencias (prioridad de acceso, éxito reproductivo, etc.); pero como en condiciones de libertad resultaba difícil el establecimiento de esta segunda perspectiva, se volvió a utilizar la descripción interaccionista, aunque se asumiera la relación entre ambos puntos de vista (Rowell, 1974).

La dominancia pretendia ser una herramienta que permitiese conocer la dirección de las interacciones sociales. Si el individuo $\mathrm{A}$ dominaba sobre el $\mathrm{B}$, cabria esperar que las interacciones agresivas partiesen de A y fueran recibidas por B en todas las ocasiones. La agresión se consideraba invariablemente unidireccional y consistente, produciéndose por lo general entre los individuos más próximos en el orden de dominancia (Bernstein y Sharpe, 1966; Sade, 1967). Rowell (1966) afirmaba, a partir de sus observaciones, que el hecho de que en la interacción el animal dominante tomase siempre la iniciativa era una visión subjetiva. Ninguna de las conductas poseía una dirección de consistencia tan absoluta que permitiera afirmar que la jerarquía, obtenida a partir de estas interacciones diádicas, fuese estable, aunque los reajustes de rango pudieran pasar inadvertidos a menos que se estudiasen especificamente.

Por otra parte, era dificil pensar que los sistemas jerárquicos de primates respondieran al modelo de uorden de picoteo" que establecía una jerarquía lineal desde el animal alfa hasta el omega, a través de sus interacciones dos a dos. Dentro de grupos reducidos de primates sería posible encontrar una jerarquía lineal en la que los individuos se ordenasen en una progresión regular dependiendo de su dominancia en cada diada (Varley y Symmes, 1966). Como se ha visto en la literatura, la unidimensionalidad, la unidireccionalidad y el rango inalterable no se correspondía con la realidad de lo que ocurre en los grupos de especies sociales. Si bien, estos criterios habían producido elementos de discusión que nos han llevado a las posiciones actuales, lo cierto es que también han deformado en un sentido reduccionista la complejidad de las dinámicas sociales.

Kawai (1958) ya había criticado la interpretación mecanicista que de la dominancia se habia hecho hasta entonces. La dominancia, como hemos visto anteriormente, no depende exclusivamente de factores relacionados con la agresión, sino también de factores dinámicos psicosociológicos que actúan en el grupo social. El rango no se podía estudiar como un conjunto de interacciones entre dos individuos, dado que el conjunto del grupo tenía una influencia determinante sobre las mismas. Estudiando el grupo Kò-Sima de macacos japoneses (Macaca fuscata), Kawai habia encontrado un sistema doble de rangos sociales: un «rango 
básico" que venía determinado por las interacciones de dos individuos, independientemente de las interferencias del resto del grupo y un "rango dependiente» determinado por las influencias del resto de los individuos sobre las interacciones diádicas (véase también Koford, 1963). El rango básico podía considerarse paralelo al rango encontrado por otros autores. Sin embargo, el rango dependiente carecia de estabilidad y cambiaba con cierta regularidad dependiendo de las situaciones sociales, de influencias externas a la interacción, tales como el parentesco con animales de alto o bajo rango, grado de intimidad, etc.

Trabajando con papiones amarillos (Papio cynocephalus), Lee y Oliver (1979) observaron que las hembras y machos jóvenes, ordenados dentro de una jerarquía completa junto con hembras adultas, tenían un rango asociado con el presumible de sus madres; pero si este rango se comparaba con el rango de los machos jóvenes, considerados únicamente entre sus iguales, no aparecía ninguna relación con el anterior. Se observó que las hembras adultas podian dominar a individuos más jóvenes en las interacciones diádicas, pero estos jóvenes podian dominar a adultas concretas, en presencia de otros adultos, generalmente sus parientes. Aunque el rango dependiente aparecía en primer lugar en la ontogenia, la relación entre rango dependiente y rango básico no era de ninguna forma estática.

Angst (1975) y De Waal (1977) aclararon mejor este punto al estudiar el desarrollo del rango en individuos jóvenes de macacos de Java (Macaca fascicularis). El rango dependiente aparecía como un estado de transición que llevaba al rango básico. Las transiciones no se producian de una manera uniforme, sino con distintos periodos de inestabilidad: los jóvenes tendian a huir ante la agresión de los adultos, pero podian también dirigir a éstos agresiones "protegidas" uniéndose en alianzas contra ellos. Posteriormente, la huida se revertía y las relaciones de sumisión también. Los jóvenes iban consolidando su capacidad agresiva y perdien- do la necesidad de solicitar ayudas, sustituyendo sus rangos dependientes por rangos básicos (Walters, 1980). Así, un macho subadulto podia comportarse como adulto en un conjunto de aspectos, mientras que en otros su comportamiento seguia siendo de joven. En el curso de todos estos reajustes de conducta el individuo va configurando su estatus (Bernstein y Sharpe, 1966). También Sade (1967) había observado que el rango de los jóvenes dependia del que tuviera su madre, de forma que al llegar a la pubertad, el rango iba dependiendo de él mismo y, sobre todo, de su capacidad física para la lucha (ver también Lee y Oliver, 1979).

Los individuos no se mantienen durante toda su vida en la tropa o grupo natal. Para Hall y DeVore (1965), tanto durante la permanencia en el grupo de nacimiento como una vez abandonado éste, los machos de babuínos oliva (Papio anubis) iban ascendiendo en jerarquia como resultado de sus interacciones agonísticas. Sin embargo, como hemos visto, al menos durante su permanencia en la tropa natal, el rango de los individuos se encuentra determinado, en primer lugar, por el rango de sus madres (Kawai, 1958; Sade, 1967; Lee y Oliver, 1979). Los hijos de hembras dominantes aprendian involuntariamente «actitudes de dominante» (Imanishi, 1960), manteniendo su rango durante mucho tiempo debido a esta asociación, hasta el momento en que se producía la ruptura.

Bernstein y Gordon ( 1980 ), estudiando los componentes sociales de la dominancia en macacos rhesus (Macaca mulatta) de distintas colonias, en condiciones experimentales, afirmaron que los rangos eran específicos de los contextos sociales y no atribuibles a los individuos. Las historias particulares de los sujetos podían servir para reducir la intensidad de los conflictos, pero no siempre determinaban sus rangos si se colocaba a estos individuos en distintos espacios. Los rangos pudieran asumirse como una consecuencia de las interacciones en contextos muy específicos, influidos por factores sociales como el número y posición de otros anima- 
les. Los primates pueden aprender y modificar sus conductas como una función de la experiencia pasada, por extinción o reforzamiento de dichas conductas respecto a otros individuos y como proyección de conflictos anteriores (Rowell, 1966; Hinde, 1976a; Hinde y Stevenson-Hinde, 1976). Un individuo puede ser dominante o sumiso dependiendo de su percepción del ambiente social. Si el ambiente cambia, el cambio puede influir decisivamente en el individuo y «... esto puede representar la situación filogenética y ecológica en la cual las habilidades cognitivas de los monos rhesus son más pertinentes" (Bernstein y Gordon, 1980, pág. 1039). De forma contraria a la opinión de Sade ( 1967 ), la dominancia depende de la experiencia pasada, hasta el punto de que la situación de un momento dado viene determinada por todas las experiencias que la han precedido (Rowell, 1966). La dominancia de ese momento no hace más que reflejar un proceso ontogénico gradual en el que se hallan implicados multitud de condicionantes (Noë et al., 1980). $\mathrm{Si}$ bien, el rango dependiente de los individuos pudiera ser consecuencia sheredada" a partir del rango de las madres, la base particular de futuras alianzas o antagonismos no está clara (Varley y Symmes, 1966), dado que, incluso para el rango básico, el problema consiste en conocer qué conductas entre dos individuos y en qué medida pueden estar o no influidas por el resto del grupo (Richards, 1974).

Ya Maslow (1936a) había observado el surgimiento de algunas conductas nuevas que parecían impredecibles, por ser una consecuencia del tamaño del grupo y la formación de algunas alianzas entre animales subordinados que derrotaban a los considerados dominantes. En cualquier circunstancia, los primates pueden cambiar sus posiciones relativas mediante la utilización de estas estrategias, uniendo sus fuerzas de forma que pudiesen elevar su rango en el grupo, al tiempo que hacían bajar el estatus relativo de sus oponentes. Aunque los individuos subordinados tuviesen más problemas en for- mar estas alianzas con otros subordinados, su contribución al balance de fuerzas dentro del grupo era efectiva (Varley y Symmes, 1966). DeVore (1965) encontró que en babuínos existía una jerarquía central y que los machos pertenecientes a ella se apoyaban entre sí cuando alguno resultaba amenazado por un individuo periférico, «independientemente de que éste fuese más joven y fuerten (pág. 91 5 ). Southwick y Siddiqui (1967) recalcaron el papel que juega la tradición social en la dominancia. Una vez que esta dominancia se encuentra establecida, la tradición puede ser suficiente para mantener esa estabilidad, aunque el macho dominante se encuentre mermado físicamente. Si el macho no pudiese defender su posición, cabría pensar en una «aceptación voluntaria» del resto del grupo para mantenerla. De cualquier forma, el medio por el que se establecen los sistemas de dominación, su desarrollo y mantenimiento no se encuentran tan bien documentados como la estructura de los mismos. Puede que los individuos diferencien, no ya sus propios aliados o antagonistas en la edad adulta, sino que cuando eran jóvenes, además de percibir el grado de dominancia de sus madres respecto a los demás, pudieran discriminar el rango de los aliados o antagonistas de sus madres (Walters, 1980). Los encuentros agresivos pueden estar influidos por interacciones triádicas (Krummer, 1967) y poliádicas. El pedir ayuda a un tercer animal no relacionado directamente con el conflicto y el éxito en la petición, puede decidir el encuentro y el rango futuro de los participantes (Struhsaker, 1967; Packer, 1977), pero depende, entre otros factores, de características idiosincráticas, es decir, de la habilidad individual para pedir ayuda. Las alianzas se ven más en la tendencia a ayudar a un individuo atacado que en la ayuda prestada al agresor (Varley y Symmes, 1966 ), dado que, por otra parte, cuando un agresor entra en conflicto con un oponente, un tercer individuo de rango más alto que dicho oponente no tendría razones para ayudar al agresor. Incluso, podria ayudar al oponente para proteger su propia posición, máxime si 
entre ellos existe alguna relación de parentesco. Sólo aquellos individuos cuyo rango fuese inferior al del agresor podrían beneficiar su propio estatus ayudando a ese agresor (Walters, I980, desde un punto de vista evolutivo).

De todo lo visto en esta sección se desprende que la jerarquía no es estable en ningún grupo y menos si la consideramos en condiciones naturales. La dominancia depende de innumerables procesos, entre los que se puede destacar el valor del aprendizaje, es decir, las jerarquías de dominancia no se mantienen inalterables, sino que se van modificando en los grupos como consecuencia de la acción del individuo a lo largo de su ontogenia y en relación con las ontogenias de los demás. Quizá, como resumen de esta idea, puede citarse el trabajo de Struhsaker (1967) sobre monos verdes (Cercopitbecus aethiops). Struhsaker observó una fuerte tendencia a la jerarquía lineal con unos pocos triángulos y relaciones probabilisticas (véase Dawkins, 1976, e Hinde, 1977). Parece deducirse que en otras especies, aunque sus estructuras sociales difieran de las de los monos verdes, los individuos organizan sus estrategias con la intención 'de alterar el statu quo que favorece a los individuos de mayor rango, los cuales organizan las suyas propias para mantener esta diferenciación (Peláez, 1982a).

Si la jerarquía no es estable resulta fácil cuestionar la idea de unidireccionalidad de la interacciones. El que un individuo $A$ sea dominante sobre $\mathrm{B}$ no significa que todas las interacciones tengan que llevar la dirección $\mathrm{A}-\mathrm{B}$, sin posibilidad de que algunas se produzcan en la dirección contraria. Así, Rowell (1966 y 1967) midió la frecuencia en que unos patrones de conducta se producian en la forma más consistente ("índice de consistencia») en una dirección de la interacción. "La relación de los individuos no es estática ni definida de una vez por todas en el primer encuentron (Rowell, 1966, página 430). Los individuos intercambian conductas, pero el intercambio podría variar dependiendo (a nivel de la diada) de la edad, salud, ciclos reproductivos, etcétera. Aunque dentro de cada diada los mismos individuos no solian manifestar conductas agresivas y sumisivas ( $\mathrm{Ri}$ chards, I974), esto podía deberse a la estabilidad de la dominancia en ese momento, pero no significa que siempre tuviese que ser así. De hecho, se observa que el mayor número de interacciones agonísticas se producen entre individuos de rango semejante (Bernstein y Sharpe, 1966, y la misma Richards, 1974). El orden de dominación aparece más inestable entre individuos próximos en el rango, mostrando períodos de «dominancia inconsistente" (Hausfater, 1975) en los que el intercambio de interacciones en la diada poseía una dirección menos definida.

\section{EL VALOR DEL CONCEPTO DE DOMINANCIA}

La dominancia se ha venido utilizando como una "taquigrafía descriptiva» para caracterizar unas interacciones entre dos individuos, dado que uno de los participantes parecía llevar la iniciativa o ganaba en los encuentros agonísticos (Noë et al., 1980). Un orden jerárquico basado exclusivamente en las observaciones de un tipo de conducta, o varios órdenes jerárquicos basados cada uno en diferente criterio(s), considerando exclusivamente quién hace a quién qué, tiene un valor meramente descriptivo (Hinde, 1974).

Pero la descripción de la estructura social en estos términos nos proporciona información limitada, si no tergiversada, de la realidad. "Hay que salvaguardarse de argumentos viciosos del tipo de que si A picotea a B, el primero tiene una posición superior en la jerarquía de dominancia» (Hinde, 1974, pág. 349). Las interacciones se refieren a dos o más individuos y engloban muchos tipos de conductas. No obstante, si los individuos se conocen entre sí, la naturaleza de las interacciones puede transformarse en el tiempo, como consecuencia de las experiencias pasadas y quizá por las expectativas de las interacciones futuras (ver tam- 
bién páginas anteriores). Es decir, las interacciones se producen en un espacio temporal en el que se establecen relaciones sociales entre los individuos.

Cuando hablamos de relaciones nos estamos refiriendo a ese conjunto de interacciones temporales entre individuos que se reconocen entre si por formar parte de un mismo grupo social (ver Hinde, 1976a, 1976b, 1974 y 1978; Hinde y Stevenson-Hinde, 1976). Al mismo tiempo, las interacciones dependen del tipo de relaciones que los individuos tienen entre sí. Si queremos que el concepto de jerarquía sea útil a un nivel aclaratorio y no meramente descriptivo hay que asumir que la posición de los individuos en las jerarquías viene determinada por las relaciones entre ellos, relaciones que pueden transportar muchos tipos de comportamientos.

La distinción entre el nivel descriptivo y explicativo es de máxima importancia. El primero no permite conocer el conjunto de razones que determina que en las interacciones sociales se produzcan unas conductas y otras no. No nos permite conocer el porqué de que el dominante controle las conductas, supuestamente, más que el subordinado. Si presuponemos que el dominante, por la misma definición, se encuentra en condiciones de obligar a cumplir el conjunto de condiciones que impone a los subordinados, obligación y fuerza son criterios que coinciden con el principio agonístico de la dominancia. Pero si, como ocurre en multitud de ocasiones, la agresión no se produce, el valor predictivo de la dominancia desaparece. Esto ha llevado a que a nivel descriptivo se establezcan distintas jerarquias de dominancia, dependiendo de los distintos criterios utilizados y a que a nivel explicativo se pretenda hacer depender la dominancia del animal que, en último lugar, decida la prioridad de la interacción (Noë et al., 1980).

Cuando se producen observaciones (generalmente, no muy descritas en la literatura) como el caso de los intercambios de alimento entre chimpancés (Pantroglody- tes) relatados por Nishida (1970), se ve que el animal dominante no tenia necesariamente que llevar la iniciativa, sino limitarse a controlar los acontecimientos. En este caso, el nivel descriptivo de la jerarquía no nos da ninguna información sobre estos hechos: el que el animal dominante sólo sea un mero controlador de los acontecimientos sociales responde a razones, más profundas.

Hemos visto, en la primera sección, que los criterios utilizados para establecer un rango de dominancia eran o se encontraban relacionados con conductas agonísticas. Cuando se establecian otros criterios, no siempre se producía una correlación con la jerarquía ya obtenida, máxime si estas correlaciones se buscaban en otras especies o incluso dentro de la misma especie pero en grupos que pertenecían a distintos hábitats. Si hay varios criterios con los que se puede establecer una jerarquía (Syme, 1974), chasta qué punto es útil el concepto de dominación? La respuesta ha venido en dos direcciones: la primera es que los problemas de interpretación han surgido como consecuencia de considerar a la jerarquía como un todo absoluto; la segunda, porque se entiende que del concepto de dominancia se ha hecho una amalgama.

La jerarquía definida mediante distintos criterios no puede dar ninguna información sobre la estructura social. Por tanto, el concepto de dominancia «es útil de forma explicativa sólo en la medida en que las diferentes maneras de apreciarla correlacionen entre sí) (Hinde, 1977, página 350 ). Sólo en este caso, la dominancia tiene la naturaleza de una variable intermedia con capacidad de predicción y posible valor explicativo conforme a la frecuencia y/o dirección de un amplio rango de distintas conductas en las interacciones sociales (Richards, 1974; Syme, 1974; Hinde, 1974, 1976 y 1978; Walters, ı 980; Noë et al., I980). Cada una de las distintas conductas serían las variables dependientes con que se aprecia la dominancia, ya que, en cualquier caso, carecería de sentido sustituir criterios como lucha, espulgamiento, etcétera, por el de 
dominancia. Si ésta se establece a partir de una única variable dependiente «es una mera forma de describir los datos con otras palabras» (Hinde, 1978, pág. 28).

La utilización del término de dominación se convierte, así, en una herramienta que economiza un número de variables dependientes en función de una variable intermedia, consiguiendo tal ahorro en la descripción que introduce un primer paso explicativo de la estructura social.

Estudiando la distribución de espulgamiento entre hembras adultas de babuínos chacma (Papio ursinus) y monos verdes (Cercopithecus aethiops), Seyfarth (1976 y 1980 , respectivamente), encontró que las hembras de cada grupo podían ordenarse en una jerarquía de dominancia lineal que predecía la dirección, pero no las frecuencias de las interacciones agonísticas. No aparecía ninguna indicación de que las frecuencias de las conductas agresivas entre las diadas estuviesen relacionadas con la posición de los individuos en la jerarquía de dominancia. Por otra parte, se podía comprobar que cuanto mayor era la diferencia entre los rangos relativos de dos individuos menos frecuentes resultaban las interacciones agonísticas entre ellos, pudiendo ocurrir que el individuo alfa fuese el que menos agresión manifestase, "como si la agresión fuera una necesidad de afirmar las categorías" (Hinde, 1977, pág. 353), produciéndose el mayor número de episodios agonísticos entre aquellos individuos cuya relación era más inestable (Bernstein, 1970). Como consecuencia, los hallazgos de Seyfarth parecen razonables.

Generalmente, se ha producido una complicada amalgama en la literatura como consecuencia de los distintos métodos empleados para establecer una jerarquia de dominancia. Estos métodos han consistido en la medición de las frecuencias de las interacciones sin tener en cuenta, en muchos casos, las relaciones de los individuos que intercambian sus conductas. Un ejemplo puede ser aclaratorio de este problema: supongamos que tenemos un grupo de tres individuos A, B y C. La frecuencia de las interacciones de una determinado tipo entre $\mathrm{B}$ y $\mathrm{C}$ pueden ser de 40 realizadas por $B$ y 25 por $C$, por lo que $B$ sería dominante sobre $C$. Las interacciones entre $A$ y $B$ son de una frecuencia de is a favor de $A$ y de $s$ a favor de B, por lo que A seria dominante sobre $\mathrm{B}$. Por último, las frecuencias entre A y C son de ro para A y 7 para C, resultando dominante $A$ sobre $C$. Sin embargo, si sumamos las frecuencias de cada una de las interacciones para cada individuo, vemos que $A$ hace 2 s ( $B$ más C), $B$ realiza 35 ( $A$ más $C$ ) y $C$ ha hecho 32 (A más B).

Estos resultados podrían interpretarse de distintas formas: A podía ser el animal más alto en la jerarquía de dominancia, ya que en cada diada domina, respectivamente, a $B$ y a $C$, mientras que $C$ sería el animal de rango más bajo por resultar dominado en cada diada por A y por B. $\mathrm{La}$ otra interpretación vendria de considerar las frecuencias totales de las interacciones en el conjunto del grupo. B resultaría ser el individuo de mayor rango, ya que su frecuencia es la más elevada (35) y A sería el de rango más bajo, como resultado de su menor frecuencia (25). Evidentemente, estos resultados tienen distinta interpretación $y$, por tanto, distinto valor explicativo, que se complicaría en el caso de utilizar más de un tipo de conducta. Por otra parte, parece evidente la necesidad de utilizar métodos distintos de los interactivos para encontrar el rango de dominancia que nos proporcione una información suficiente sobre la estructura social del grupo que estudiamos. Estos problemas permanecen sin resolver.

\section{Relaciones \\ de dominancia/subordinación entre diadas}

Para poder llegar a establecerlas se necesita que exista un cierto número de diadas, que cada diada posea una relación múltiple con interacciones complementarias (un participante actúa de forma dis- 
tinta que el otro pero complementando la interacción) y que en cada una de las diadas posibles exista un individuo que realice más que el otro cada una de las conductas. Es necesario tener en cuenta que al hablar de relaciones, el concepto no se refiere a la cantidad de conductas que los individuos muestran, sino al resultado del balance entre las conductas de uno y otro en la diada para el conjunto de las interacciones que consideremos.

La agresión podía ser un criterio, pero, como hemos visto en Bernstein (1970), no siempre el individuo más dominante exhibe mayor agresión (ver también Kaufmann, 1967; Tokuda y Jensen, 1969; Reynolds y Luscombe, 1969), dado que la dirección de complementaridad puede haberse establecido en los primeros encuentros mediante acciones agresivas y las conductas que ahora se manifiestan serian un resultado de aquéllas, con la agresión ausente (Richards, 1974; Seyfarth, 1976; Hinde, 1978)

Sólo si estas condiciones se cumplen en la relación diádica, pueden establecerse las relaciones de dominancia/subordinación como una variable intermedia que economice y prediga la dirección de las interacciones entre ambos individuos.

\section{Rango de dominancia}

El concepto de rango de dominancia es unificador, descriptivo y puede utilizarse con un cierto valor explicativo si a partir de un conjunto de variables dependientes se obtienen correlaciones entre ellas, de forma que "rango de dominancia" pueda considerarse también como una variable intermedia economizadora. Sólo de esta forma, el rango de dominancia puede ser útil en la explicación de la estructura social (Hinde, 1974). Sin embargo, el mismo Hinde (1978) consideró excesiva esta restricción, volviendo a replantear esta idea: el establecimiento de un orden jerárquico puede resultar de utilidad para algunos aspectos de la conducta que no se encuentren correlacionados con él. Sería el caso de aquellos individuos que en la diada se comportan de una forma particular (conforme a conductas no correlacionadas), dependiendo no de sus posiciones jerárquicas individuales, sino de las diferencias que existen entre ellos debido a sus posiciones relativas en ese rango. Esto explicaría las particularidades en las conductas de dos individuos con rangos adyacentes (Hausfater, 1975). En los casos de Seyfarth (1976 y 1980), la aparente contradicción podria explicarse por el hecho de que las hembras que más agresión exhibían entre diadas no eran dominantes en el grupo completo. Hinde (1978, pág. 31) concluyó que las hembras de Seyfarth no sólo respondían (por espulgamiento) de una forma particular ante las hembras de mayor rango, sino que graduaban sus respuestas dependiendo de la medida en que ese rango era superior.

\section{FUNCION DE LA DOMINANCIA SOCIAL}

Si sabemos que la dominancia es una variable intermedia que economiza la utilización de un conjunto de variables dependientes, resulta dificil adscribir a la dominancia social una función concreta. Sin embargo, la dominancia ha estado asociada en la literatura con determinadas ventajas para el grupo y/o para los individuos considerados como dominantes dentro del mismo. De la revisión anterior se deduce ya el valor relativo de esta asociación.

Southwick y Siddiqui ( 1966 ) hicieron una revisión de los papeles de los machos dominantes en distintos grupos de primates del Viejo Mundo. Los machos dominantes protegían al grupo de las amenazas externas, mediaban en las agresiones y estabilizaban las relaciones sociales en el grupo, mantenían el territorio, dirigían a los grupos en los movimientos y poseían una ventaja en la selección de pareja. El desarrollo de un orden estable de dominancia evitaba progresivamente las luchas continuas y los castigos (Richards, 1974). Los machos dominantes tendrían la fun- 
ción de amortiguar o reducir estas interacciones agresivas que pudieran resultar maladaptativas para el conjunto del grupo (Bernstein y Sharpe, Bernstein, I968; Tokuda y Jensen, I968; Saayman, 1971). La estabilidad jerárquica se considera beneficiosa para el grupo y un factor "deseable" (Walters, 1980) para cada uno de sus miembros. No obstante, en el único caso en el que cabría esta última explicación sería si admitiésemos una selección a nivel de grupo. Si, por el contrario, la selección se produce a nivel de individuo, la explicación puede venir de considerar a la dominancia como una estrategia evolutivamente estable (ver Maynard Smith y Price, I 973). Es decir, como una solución considerando las distintas opciones que el individuo puede adoptar en función de las opciones de los demás (Walters, 1980). De cualquier forma, la visión generalizada de que la jerarquia de dominancia reduce conflictos, puede haberse producido a partir de la comparación establecida entre la violencia observada en grupos de nueva formación y la posterior disminución de las luchas cuando el grupo se estabiliza (Rowell, I974).

A los individuos dominantes en el grupo se les ha asignado la facultad de ciertas tomas de decisión en cuanto al inicio de un viaje, al orden de progresión y otras actividades (Bernstein y Sharpe, 1966; Varley y Symmes, 1966; Saayman, I971; Kummer, I968). Estos aspectos de la conducta de los dominantes, junto con la facultad de finalizar las interacciones agonisticas, han sido los argumentos principales para otorgar el rol de «lider" a estos individuos (Gartlan, I968; Crook, 1970; Bernstein y Sharpe, 1966). Aunque en hamadríades (Papio bamadryas) los machos lideres suelen obligar a sus hembras a que les sigan (Kummer, 1968a; Kummer et al. 1970), no deja de ser una particularidad: en otros casos la dirección de progresión durante los traslados parece estar dirigida no por los individuos de mayor rango, sino por los más viejos y experimentados (Rowell, 1974).

Otro aspecto intimamente relacionado con la jerarquía de dominancia ha sido el reproductivo (Bernstein y Sharpe, 1966; Hall y DeVore, I965; Lee y Oliver, 1979; Hausfater, I975, etc.). Una alta dominancia traería consigo un aumento de la descendencia potencial (Lee y Oliver, 1979). En macacos rhesus (Macaca mulatta), Wilson ( 198 I) ha encontrado que las hembras de alto rango poseían un mayor éxito reproductivo relacionado con una menor tasa de mortalidad infantil. Aunque las hembras de menor rango estuviesen implicadas en igual medida que las de alto rango en actividades copulatorias, las primeras sufrirían una mayor pérdida de hijos antes y después del parto. Cualquier ventaja reproductiva, concluye Wilson, no es un resultado de la competición sexual (?), al menos en rhesus. Hay que distinguir entre éxito reproductivo y éxito en la formación de pareja. Para Witt et al. (198I) el rango del macho determina la proporción y el número absoluto de descendientes a partir de hembras que tienen también alto rango. El acceso a las hembras vendria determinado tanto por el rango básico como por la capacidad de formar coaliciones. La exclusión de los competidores subordinados en interacciones diádicas proporciona al ganador un mayor éxito reproductivo, pero la formación de coaliciones también produce un beneficio al conjunto de los coaligados. Por el contrario, para Smith (1981), el argumento de que las jerarquías de dominancia han evolucionado por selección sexual ha creado la idea de que los individuos (machos y hembras) de alto rango producen un número desproporcionado de descendientes. Al parecer, el éxito reproductivo en rhesus se encuentra muy correlacionado con rango, pero, aunque no hay evidencia, es muy posible que las posiciones en los rangos sean consecuencia y no causa de ese éxito reproductivo. En especies que son estacionales, la jerarquía se forma independientemente de la actividad reproductora. En estos casos, la asociación que se encuentre entre dominancia y éxito reproductivo pudiera indicar una ventaja selectiva para los individuos de alto rango. 


\section{Referencias}

ANGST, W.: «Basic data and concepts on the social organisation of Macaca fascicularis». Primate Beba. 4: 325-388. 1975 .

ARDREY, R.: Tbe Territorial Imperative. Antoney Blond. Londres, 1967.

BERNSTEIN, I. S.: «Social status of two hybrids in a wild troop of Macaca irus». Folia primatol. 8: 121-131. 1968.

Bernstein, I. S.: «Primate status hierarchies Rosenblum, L. A. (Ed.): Primate Bebavior. Vol. 1. 7 I-109. Academic Press. Nueva York, 1970.

Bernstein, I. S., y Gordon, T. P.: "The social component of dominance relationships in rhesus monkeys (Macaca mulatta)". Anim. Bebav. 28. (4): 1033-1039. 1980.

Bernstein, I. S., y SHARPE, L. G.: «Social roles in a rhesus monkey group». Bebaviour. 26. (1,2): 91 1-104. 1966.

CARPENTER, C. R.: "Tentative generalizations on the grouping behavior of non-human primates". En Gavan, J. A. (Ed.): The Non-Human Primates and Human Evolution. 91, Wayne Univ. Press. Detroit, 1954

Chance, M. R. A.: «Social structure of a colony of Macaca mulatta». Brit. J. Anim. Bebav. 4: I-13. 1956.

CHANCE, M. R. A.: «Attention structure as the basis of primate rank orders». Man. 2: $503-518$. 1967.

Chance, M. R. A.: «Attention structure and social organization». En ChIvers, D. J., y Herber, J. (Eds.): Recent Advances in Primatology. Vol. I, 93-98. Academic Press. Nueva York, 1978.

Chance, M. R. A., y Jolly, C. J.: Social Groups of Monkeys, Apes and Man. E. P. Dutton. Nueva York, 1970.

Chance, M. R. A., y Larsen, R. R.: The Social Structure of Attention. John Wiley \& Sons. Londres, I 976.

Crawford, M. P.: "The social psychology of the vertebrates". Psychol. Bull. 36: 409-445. 1939.

Crook, J. H.: «Social organization and the environment: aspects of contemporary Social Ethology». Anim. Bebav. I8: 197-209. 1970.

DAWKINS, R.: «Hierarchical organization: a candidate principle for Ethology». En BATESON, P. P. G., y Hinde, R. A. (Eds.): Growing Points in Ethology. 7-54. Cambridge Univ. Press. Cambridge, 1976.

DEVORE, I.: "Male dominance and mating behavior in babbons". En BEACH, F. A. (Ed.): Sex and Behavior. 266-189. John Wiley \& Sons. Nueva York, 1965 . (Traducción castellana Siglo XXI, Méjico).

EMORY, G. M.: «Aspects of attention, orientation and status hierarchy in mandrillus (M. sphinx) and gelada babbons (Theropitbecus gelada)». Bebaviour, s9: 70-87. 1976.

GaRTLAN, J. S.: «Structure and function in primate society». Folia primatol. 8: 89-120. 1968.

HAll, K. R. L., y DEVORE, I.: «Baboon social behavions. En DeVoRE, I. (Ed.): Primate Behavior: Field Studies of Monkeys and Apes. 53-1 10. Holt, Rinehart \& Winston. Nueva York, 1965.

Hausfater, G.: Dominance and Reproduction in Baboons (Papio cynocepbalus). A Quantitative Analysis. Contributions to Primatology. Vol. 7. Karger. Basel, 1975.

Hinde, R. A.: Biological Bases of Human Social Bebavior. McGraw Hill. Nueva York, 1974. (Traducción castellana, Siglo XXI. Méjico, 1977).

HINDE, R. A.: «Interactions, relationships and social structure». Man. $11: 1-17.1976 a$.

HindE, R. A.: «On describing relationships». J. Cbild Psycbol. Psycbiat. 17: 1-19. 1976b.

HINDE, R. A.: «Dominance and role-two concepts with dual meanings». J. Social Biol. Struct. 1: $27-38.1978$.

Hinde, R. A., y STEvenson-HINDE, J.: "Towards understanding relationships: dinamic stability». En Bateson, P. P. G., y Hinde, R. A. (Eds.): Growing Points in Etbology. 45 1-479. Cambridge, Univ. Press. Cambridge, 1976.

ImANISH, K.: «Social organization of subhuman primates in their natural habitats». Curr. Antbrop. 1: 393. 1960.

KAUFMANN, J. H.: "A 3-year study of mating behaviour in a free-ranging band of rhesus monkeys». Ecology. 46: 500-912. 1969 .

KaUfmanN, J. H.: "Social relation of adult males in a free-ranging band of rhesus monkeys". En Altmann, S. A. (Ed.): Social Communication Among Primates. 73-98. Univ. Chicago Press. Chicago, 1967.

KAWAI, M.: "On the system of social ranks in a natural troop of Japonese Monkeys. I. Basic rank and dependent rank». Primates. I: I I I-130. 1958.

KOFORD, C.: «Ranks of mothers and sons in bands of rhesus monkeys». Science. 141: 356-357. 1963.

Kummer, H.: "Tripartite relations in Hamadryas Baboons". En Altmann, S. A. (Ed.): Social Communication Among Primates. 63-71. Univ. of Chicago Press. Chicago, 1967.

Kummer, H.: Social Organization of Hamadryas Baboons. Bibl. Primatol. Vol. 6. Karger. Basel, 1968.

KUMmer, H.; GOETz, W., y ANGST, W.: «Cross-species modification of social behavior in baboons». En NAPIER, J. R., y NAPIER, P. H. (Eds.): Old World Monkeys: Evolution, Systematics and Bebaviour. 35 1-363. Academic Press. Londres, 1970.

LEE, P. C., y Ol.Iver, J. I.: «Competition, dominance and the acquisition of rank in juvenile yellow baboons (Papio gynocepbalus)". Anim. Bebav. 27 (2): 576-58 1979.

MASLOW, A. H.: "The role of dominance in the social and sexual behavior of infra-human primates. IV. The determination of Hierarchy in pairs and in a groupn. J. Genetic. Psycbol. 49: 161-198. 1936a.

MASLOW, A. H.: "The role of dominance in the social and sexual behavior of infra-human primates. I. Observations at Vilas Park Zoow. J. Genet. Psycbol. 48: 261-277. $1936 \mathrm{~b}$.

Maslow, A. H.: «Dominance quality and social behavior in infra-human primates». J. Soc. Psychol. 2: 31 3-324. 1940. 
MASON, W. A.: "The effects of social restriction on the behaviour of thesus monkeys. III. Dominance Tests». J. Comp. Pbysiol. Psycbol. s4: 694-699. 1961.

Maynard Smith, J., y Price, G. R.: "The logic of animal conflict». Nature. 246: is-18. 1973.

NishidA, T.: "Social behavior and relationship among wild chimpanzees of the Mahali mountains". Primates. II: $47-87.1970$.

NOE, R.; WAAL, F. B. M. DE, y HoOFF, J. A. R. A. M., vaN: "Types of dominance in a chimpanzee colony». Folia Primatol. 34 (1-2) 90-1 10. 1980.

PACKER, C.: «Reciprocal atruism in Papio anubis». Nature. 26\%: 441-443. 1977.

Pelaez, F.: «Estructura social y conductas de saludo en dos especies de babuinos». Estudios de Psicología 10: 34-52. $1982 \mathrm{a}$.

PelAeZ, F.: "Greeting Movements Among Adult Males in a Colony of baboons: Papio bamadryas, P. cynocephalus and their Hybrids". Primates. 23 (2): 233-244. 1982 b.

Pollock, J. I.: «Female Dominance in Indri indri». Folia Primatol. 31: 143-164. 1979.

REYNOLDS, V., y LuSCOMBE, G.: «Chimpanzee rank order and the function of displays». En CARPENTER, C. R. (Ed.): Proc. 2nd. Congr. Primat. Atlanta, 1968. Vol. 1. 8 I-86. Karger. Basel y Nueva York, 1969.

RichardS, S. M.: "The Concept of Dominance and Methods of Assessment». Anim. Behav. 22: 91 4-930. 1974. ROWELl, T. E.: «Hierarchy in the Organization of a Captive Baboon Group». Anim. Behav. 14: 430-443. 1966.

Rowel.L, T. E.: "A Quantitative Comparison of the Behaviour of a Wild and a Caged Baboon Group". Anim. Bebav. 15: 499-509. 1967.

ROWELL, T. E.: "The concept of social dominance». Bebav. Biol. 11 : I31-1 54. 1974.

SAAYMAN, G. S.: «Behaviour of the adult males in a troop of free-ranging chacma baboons (P. ursinus)" Folia Primatol. 15: 36-57. 1971.

SADE, D. S.: «Determinants of Dominance in a Group of Free-Ranging Rhesus Monkeys». En ALTMANN, S. (Ed.): Social Communication Among Primates. 99-1 14 . Univ. of Chicago Press. Chicago, 1967.

Seyfarth, R. M.: «Social relationships among adult female baboons». Anim. Behav. 24: 917-938. 1976.

SEYFARTH, R. M.: "The distribution of grooming and related behaviours among adult female vervet monkeys». Anim. Bebav. 28 (3): 798-8 I 3 . 1980.

SIMONDS, P. E.: "The bonnet macaque in South India». En DEVORE, I. (Ed.): Primate Behaviour: Field Studies of Monkeys and Apes. 175-196. Holt, Rinehart and Winston. Nueva York, 1965.

SMITH, D. G.: "The association between rank and reproductive success of male rhesus monkeys". $A m$. J. Primatol. I (1): 83-90. 1981.

Southwick, C. H., y SIDDIQI, M. R.: "The role of social tradition in the maintenance of dominance in a wild thesus groupm. Primates. 8: 341-353. 1967.

STRUHSAKER, T. T.: «Social structure among Vervet monkeys». Behaviour. 29: 83-121. 1967.

SYME, G. J.: "Competitive orders as measure of social dominance». Anim. Bebav. 22: $931-940.1974$.

TOKUDA, K., y JENSEN, G. D.: «Determinants of dominance hierarchy in a captive group of pigtailed monkey (Macaca nemestrina)». Primates. 10: 227-236. 1969.

TORRes DE AssumpÇAO, C., y DEAG, J. M.: «Attention Structure in Monkeys. A Search for a Common Trend». Folia Primatol. 31 : 28 5-300. 1979.

VARLEY, M., y SYMMES, D.: «The hierarchy of dominance in a group of macaques». Bebaviour. 27: 54-75. 1966.

WALL, F. B. M. DE: «The organization of agonistics relations within two captive groups of Java Monkeys (Macaca fascicularis)». Z. Tierpsycbol. 44: 22 25-282. 1977.

WALTERS, J.: «Interventions and the Developement of Dominance Relationships in Female Baboons». Folia Primatol. 34: 6I-89. 1980 .

WILSON, M. E.: "Social dominance and female reproductive behaviour in rhesus monkeys (Macaca mulatta)". Anim. Bebav. 29 (2): 472-482. 1981.

WITT, R.; SCHMIDT, C., y SCHMITT, J.: «Social Rank and Darwinian Fitness in a Multimale Group of Barbary Macaques (Macaca syluana Linnaeus, 1758). Dominance Reversals and Male Reproductive Success). Folia Primatol. 36 (3-4): $201-211.1981$.

Zuckerman, S.: The Social Bebavior of Monkeys and Apes. Kegan Paul. Londres, 1932.

\section{Resumen}

La dominancia social ba sido un concepto ampliamente debatido en la literatura conductual. Este artículo presenta una revisión de distintos aspectos: la unidireccionalidad, unidimensionalidad, valider del concepto $y$ función de la dominancia se discuten desde una perspectiva primatológica. Desde un punto de vista descriptivo se diferencian las relaciones de Dominancia/Subordinación entre diadas y rango de dominancia. Ambos conceptos son útiles si, en cada caso, son variables intermedias que aborran relaciones entre otras variables de la conducta social, pudiendo introducir, en este caso, a la explicación de la estructura social. 


\section{Estudios}

\section{Summary}

Social dominance bas been a bardly discussed concept in bebavioural literature. From a primatological perspective this article is a revien of different aspects related to dominance: unidireccionality, unidimensionality, concept's value and function of dominance are discussed. Dominance/Subordinance relations witbin dyads and dominance rank are differentiated from a descriptive point of view. Botb concepts, in each case, are useful so far as provide economy of relations among social bebaviour variables, introducing in this way a first stage in social structure explanation. 